\title{
Good Work Deserves Good Pay: A Quality-Based Surplus Sharing Method for Participatory Sensing
}

\author{
Shuo Yang ${ }^{\dagger}$, Fan $\mathrm{Wu}^{\dagger}$, Shaojie $\mathrm{Tang}^{\ddagger}$, Xiaofeng $\mathrm{Gao}^{\dagger}$, Bo Yang ${ }^{\dagger}$, Guihai Chen ${ }^{\dagger}$ \\ ${ }^{\dagger}$ Shanghai Key Laboratory of Scalable Computing and Systems, Shanghai Jiao Tong University, China \\ ${ }^{\ddagger}$ Department of Information Systems, University of Texas at Dallas, USA \\ $\dagger\{$ wnmmxy, wu-fan, gao-xf, bo.yang, gchen $\} @$ sjtu.edu.cn; ${ }^{\ddagger}$ tangshaojie@gmail.com
}

\begin{abstract}
Participatory sensing has become a novel and promising paradigm in environmental data collection. However, the issue of data quality has not been carefully addressed. Low quality data contributions may undermine the effectiveness and prospects of participatory sensing, and thus motivates the need for approaches to guarantee the high quality of the contributed data. In this paper, we integrate quality estimation and monetary incentive, and propose a quality-based surplus sharing method for participatory sensing. Specifically, we design an unsupervised learning approach to quantify the users' data qualities and longterm reputations, and exploit an outlier detection technique to filter out anomalous data items. Furthermore, we model the process of surplus sharing as a cooperative game, and propose a Shapley value-based method to determine each user's payment. We have conducted a participatory sensing experiment, and the experiment results show that our approach achieves good performance in terms of both quality estimation and surplus sharing.
\end{abstract}

\section{INTRODUCTION}

The rapid proliferation of smartphones has brought us an efficient and pervasive way to collect large amounts of data. On one hand, smartphones are becoming increasingly indispensable in people's daily life, not only in communication and social interaction, but also in sports, health, business, and navigation. On the other hand, most of the smartphones are programmable and embedded with various kinds of sensors, e.g., microphone, GPS, compass, accelerometer, and gyroscope. By designing specific smartphone applications, we can monitor users' surrounding environment and infer human activities. Particularly, participatory sensing [1]-[3], raised in recent years, utilizes mobile devices to gather, analyze, and share their local information, e.g., noise, pollution, and traffic information. It has been applied to various scenarios, including localization [4]-[7], indoor floorplan construction [8], [9], environmental monitoring [10], transportation and navigation [11]-[15], and photo transmission [16].

This work was supported in part by the State Key Development Program for Basic Research of China (973 project 2012CB316201), in part by China NSF grant 61422208, 61472252, 61272443, 61133006 and 61202024, in part by CCF-Intel Young Faculty Researcher Program and CCF-Tencent Open Fund, in part by the Scientific Research Foundation for the Returned Overseas Chinese Scholars, in part by Jiangsu Future Network Research Project No. BY2013095-1-10, in part by Shanghai NSF 12ZR1445000, and in part by Shanghai Pujiang Program 13PJ1403900. The opinions, findings, conclusions, and recommendations expressed in this paper are those of the authors and do not necessarily reflect the views of the funding agencies or the government.

$\mathrm{F}$. Wu is the corresponding author.

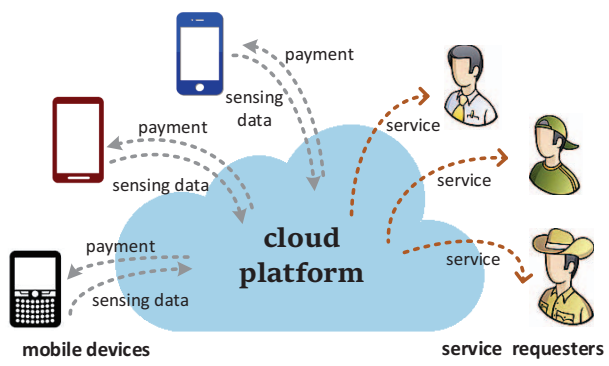

Fig. 1. Participatory Sensing Architecture

A typical participatory sensing architecture, as shown in Fig. 1, consists of three major components: a cloud platform, mobile device users, and service requesters. After releasing a sensing campaign, the platform asks part of the mobile device users to perform sensing tasks, i.e., to use their mobile devices to collect specific local information and send sensing readings back to the platform. Based on the collected sensing data, the platform gets a global view of the users' local knowledge and thus can provide certain information to the service requesters. For instance, the platform asks the mobile phone users to report their local traffic conditions. After processing and analyzing the users' reports, the platform can provide drivers with real-time traffic status, as well as congestion forecast.

Despite the novelty and potential of participatory sensing, it has exposed two critical problems. One of the problems lies on the great differences among the qualities of the users' contributed data. Since different brands of mobile devices are produced by different manufactures and are assembled with diverse series of sensors, they usually have heterogeneous sensing capabilities, resulting in varying data qualities. Besides the factors of devices, human behaviors, which are more complicated and less likely to be predicted, could also influence the data qualities. For example, some users are obedient and strictly follow the platform's instructions, while some users may deliberately contribute low quality data for their own sake. Some careless users may inadvertently contribute erroneous data by taking incorrect measurement approaches, such as putting a phone in the pocket while being asked to collect noise information. Thus, simply adopting all the contributed data can be misleading. It is of great necessity to estimate the users' data qualities, to filter out anomalous data items, and also to characterize the users' sensing behaviors. 
The other problem is how to incentivize the users to continuously participate in the sensing campaign. Since performing sensing tasks requires the mobile device users to devote their time, battery consumption, storage spaces, and computation resources, rational users, who only consider their own benefits, may not be willing to participate in the sensing campaign without proper compensation. To motivate the users' willingness on participation, the platform usually rewards each user with a certain amount of payment. Most of the existing works determine the users' payments by adopting a reverse auction model. In the auction, each user submits her self-claimed cost as her bid. Then the platform selects part of the users to perform sensing tasks and rewards each selected user with a payment no less than her bid [17]-[20]. However, these reverse auction-based methods suffer from several critical limitations in practice. First, it is infeasible to determine a user's cost for participating in a sensing campaign, since her devotion, e.g., battery consumption [21], cannot be precisely quantified by existing approaches. Second, since the users' self-claimed costs cannot reflect their qualities of contributions in a sensing campaign, determining the users' payments based solely on their bids may leaves users with chances to provide low or no effort, commonly known as "free-riding" [22]. Third, without quality regulation, collected data may suffer from uneven levels of qualities, which prevents the platform from providing reliable services to requesters and thus diminishes the utility of the platform.

With the objectives of regulating data quality and motivating the users' participation, a quality-based payment determination scheme is badly needed. To the best of our knowledge, none of the existing works take both monetary incentive and data quality into consideration. A number of researchers [17][20], [22]-[24] studied the incentive problems in participatory sensing, but did not provide a way to measure the data quality. Although Wang et al. [16] and Huang et al. [25] preliminarily investigated the issues of data quality, they did not consider the important part of monetary incentives.

In this paper, we jointly consider the problems of quality estimation and monetary incentives, and propose a qualitybased surplus sharing method, which mainly consists of two parts: (i) quality estimation module and (ii) surplus sharing module. In the quality estimation module, we present an unsupervised learning technique to estimate the users' data qualities and characterize their long-term reputations. To improve the accuracy of data analysis, we also detect and filter out anomalous users, whose sensory readings are far away from the group consensus. To determine the users' payments, we model the process of surplus sharing as a cooperative game, where the total surplus earned by the platform is based on the users' contributions. We adopt the concept of the celebrated Shapley value [26], [27] to calculate each user's surplus share. To tackle the problem of infeasibility in calculating the Shapley values, we propose an approximate Shapley value calculation algorithm. We show that the proposed surplus sharing scheme exhibits several desirable properties that indicate that a user's payment is proportional to her contribution to the sensing campaign. We also conduct an experiment to evaluate our proposed method. Our major contributions are listed as follows.

- First, we propose an unsupervised learning method to quantify the users' data qualities, and to characterize their long-term reputations based on their quality records. We also apply an outlier detection technique to improve platform's estimation accuracy.

- Second, we model the process of surplus sharing as a cooperative game, and discuss several desirable properties in designing a surplus sharing scheme. We propose a Shapley value-based surplus sharing method that satisfies our design requirements. We also present an approximate Shapley value calculation algorithm to reduce the computation complexity.

- Third, we have conducted a noise monitoring experiment for more than 12 hours, and collected over 450,000 data items. Our experiment results show that our method achieves good performance in both quality estimation and surplus sharing.

The rest of the paper is organized as follows. We first briefly review related work in Section II. In Section III, we present our system model. The quality estimation module and the surplus sharing model are presented in Section IV and Section $\mathrm{V}$, respectively. In Section VI, we conduct an experiment and evaluate our proposed method. Finally, we conclude this paper in Section VII.

\section{RELATED WORK}

The concept of participatory sensing was initialized by Burke et al. [1], after which a good number of researchers have studied various applications of participatory sensing. Azizyan et al. [4] proposed a logical localization technique based on ambient fingerprintings, e.g., optical, acoustic, and motion attributes. LiFS [5], Zee [6], and FreeLoc [7] are three different physical indoor localization methods that deploy mobile devices to track the indoor environment. CrowdInside [8] and Jigsaw [9] both leverage smartphone sensors to automatically construct indoor floor plan. PEIR [10] is a participatory sensing application that calculates personalized estimates of environmental impact and exposure based on data collected from mobile phones. SmartPhoto [16] is a smartphone-based resource-aware crowdsourcing approach for image sensing. Besides, participatory sensing has also been applied to traffic and navigation, e.g., estimating traffic delay [11], finding the most fuel-efficient routes for vehicles [12], predicting bus arrival time [13], finding on-street parking spaces [14], and automatically updating road maps [15].

Participatory sensing has also been intensively studied from the theoretical perspectives, especially based on the market model, where users are modeled as rational and are only interested in maximizing their own benefits. Lee and Hoh [17] studied the user participation problem and proposed an incentive mechanism to minimize incentive cost, while maintaining an adequate number of participants. Later, Jaimes et al. [23] extended Lee and Hoh's work to a location-based 
scenario with budget constraint. Yang et al. [18] considered both the platform-centric model and the user-centric model, and provided incentive mechanisms for them respectively. Koutsopoulos [19] modeled the participatory sensing as a reverse auction, and studied the design of optimal frugal mechanism. Zhao et al. [20] studied the online task allocation in participatory sensing with budget constraint. The most closely related works to ours are [22] and [25]. Zhang and van der Schaar [22] proposed a reputation-based protocol to incentivize users to contribute high level of effort, but they simply assumed that the users' behaviors could be classified into binary sets (i.e., good or bad) and did not provide any method to measure the qualities of the users' contributions. Huang et al. [25] proposed a quality and reputation framework for noise monitoring, but they neither eliminated anomalous users nor considered the monetary incentives.

Outlier detection [28], which has been widely studied in the field of data mining, has also been applied to sensor network to detect faulty nodes and improve sensing accuracy [29], [30]. It can be mainly classified into model-based and consensusbased. A model-based outlier detection technique requires prior knowledge of the data distribution and tends to detect data instances that deviate from the expectation. Whereas, the consensus-based protocols measure the confidence of data instances based on the group consensus and thus do not need additional data models. The consensus-based approaches can be further classified into distance-based [31] and densitybased [32], depending on which consistency metric (distance or density) is used. In this paper, we apply a distance-based outlier detection technique [31] to detect anomalous users.

Shapley value [26], [27] is a powerful tool for surplus sharing in cooperative games, where multiple players cooperate with each other to generate a surplus and the problem is to determines each player's surplus share. It has been applied to various scenarios. Misra et al. [33] studied the incentive problem in peer-to-peer scenario and proposed a fluid Shapley value approach to guarantee that each peer receives a payment proportional to its contribution. Narayanam and Narahari applied Shapley value to discover influential nodes in social networks [34]. Ma et al. [35] studied the profit sharing in ISP settlement, and presented a sharing mechanism based on Shapley value. Dong et al. [21] modeled the energy accounting as cooperative game, and provided a Shapley value-based approach to determine the energy consumption of each application in a smartphone.

\section{SyStem OVERVIEW}

In this section, we present an overview of our proposed participatory sensing architecture.

We consider a general participatory sensing scenario, where the platform's objective is to monitor an unknown environmental condition (e.g., noise, temperature, or traffic condition). To this end, mobile device users are asked to gather and share their local environmental information, which will be used by the platform to generate its estimation of real environment. Since the accuracy of the collected data may vary among

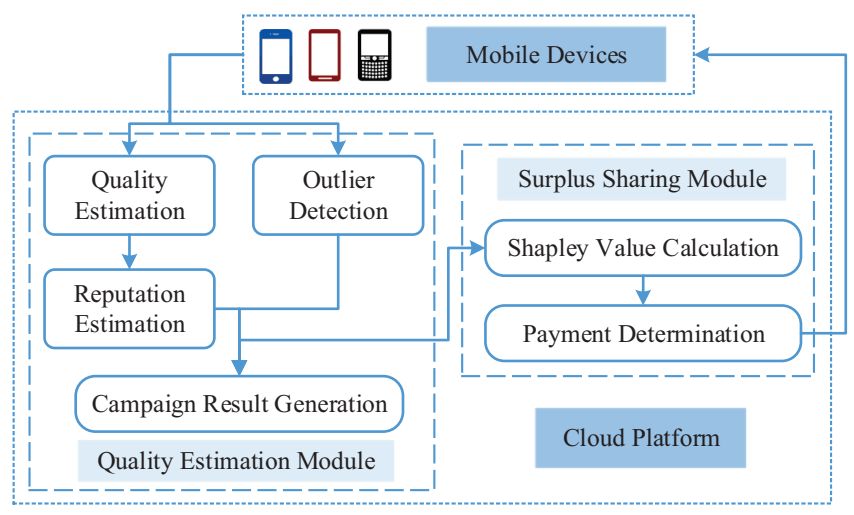

Fig. 2. System Overview

users, it is of great necessity to quantify the users' data quality, s.t., the users' contributed data will be treated differentially in producing the campaign estimation. Furthermore, the users' payments will be determined based on their data qualities.

We note that the environmental conditions may differ among distinct locations and moments. For example, the traffic conditions at two different locations may not be the same at the same time. Even at the same location, they may vary among different moments. To tackle the spatial and temporal inconsistencies, the participatory sensing campaign is divided into tasks, each of which has its specified area and period [23], [36]. The users are allowed to choose and participate in their interested tasks. For clarity of illustration, we consider the quality estimation and surplus sharing for one task in the rest of the paper.

We assume that a task (e.g., noise monitoring in a specific park) has $K$ time slots with the same duration $T$. The set of users within the region of the task is denoted by $\mathbb{N}=$ $\{1,2, \ldots, n\}$. In each time slot $k, 1 \leq k \leq K$, each user $i \in \mathbb{N}$ submits her sensing data $x_{i, k}$ to the platform, and is rewarded with payment $p_{i, k}$.

Fig. 2 shows the architecture of our proposed participatory sensing system, which primarily consists of a quality estimation module and a surplus sharing module. The quality estimation module is adopted to quantify the users' qualities and reputations, to classify the users into normal or anomalous, and to calculates the platform's estimation of the real environment. Based on the results from the quality estimation module, the surplus sharing module applies the Shapley value to determine the users' payments.

In the quality estimation module, the platform utilizes an unsupervised learning technique to estimates the users' data qualities $Q_{k}=\left\{q_{1, k}, \ldots, q_{n, k}\right\}$ in each slot $k$ without the knowledge of the ground truth, where $q_{i, k}$ represents the relative accuracy of the user $i$ 's contributed data. Although quality estimation can provide comparisons of the users' data in current slot, it neglects the users' historical behaviors and only presents a temporal view of the users' data qualities. To completely characterize the credibility of the users' data, a reputation component is introduced to aggregate each user $i$ 's 
historical quality records to quantify her reputation $R_{i, k}$ after $k$ slots. A high reputation score $R_{i, k}$ means that user $i$ has been contributing high quality data in the past slots and thus her data $x_{i, k}$ in current slot $k$ is more likely to be accurate.

To improve the accuracy of our generated estimation, we apply an outlier detection technique [31] to classify the users into two sets, i.e., a set of normal users $\mathbb{N}_{k}^{N}$ and a set of anomalous users $\mathbb{N}_{k}^{A}$, depending on whether one's sensing data is far away from the group consensus. The data contributed by the anomalous users is considered to be faulty and thus should be filtered out in the process of campaign result generation. Finally, based on the results from reputation estimation and outlier detection, we generate our real-time campaign result $\bar{x}_{k}$, which is our estimation for environmental condition.

In the surplus sharing module, we determine each user's payment by modeling the campaign as a multi-player cooperative game [37], i.e., players participate in a game and the game generates a surplus which will be divided among the players. We note that the term "cooperative" means that players can influence the total generated surplus via both cooperation and competition. In participatory sensing, the game is the sensing campaign, the players are the mobile device users, and the surplus is the platform's profit from the campaign. We assume that the platform's profit is proportional to the credibility of its generated campaign result, where the credibility of campaign result is based on the credibility of normal users $\mathbb{N}_{k}^{N}$. We analyze several desirable properties in surplus sharing and propose a Shapley value-based method. We show that the proposed method perfectly fits our design requirements. To tackle the exponential complexity of Shapley value computation, we further propose an efficient algorithm to calculate the approximate Shapley value of each user.

We summarize the frequently used notations in Table I.

TABLE I

FREQUENTLY USED NOTATIONS

\begin{tabular}{cl}
\hline \hline Notation & Description \\
\hline $\mathbb{N}, n$ & Set of users and the number of users \\
$\mathbb{S}$ & Subset of users \\
$i, j$ & User \\
$K, k$ & The number of slots and slot \\
$T$ & The duration of each slot \\
$X_{k}, x_{i, k}$ & Data set and $i$ 's data in slot $k$ \\
$Q_{k}, q_{i, k}$ & Quality set and $i$ 's quality in slot $k$ \\
$R_{i, k}$ & User $i$ 's reputation after slot $k$ \\
$\mathbb{N}_{k}^{N}, \mathbb{N}_{k}^{A}$ & Normal users and anomalous users in slot $k$ \\
$w_{k}$ & Cluster centroid in slot $k$ \\
$\bar{x}_{k}$ & Campaign result in slot $k$ \\
$p_{i, k}$ & The payment of $i$ in slot $k$ \\
$v(\mathbb{S})$ & The surplus generated by the set $\mathbb{S}$ of users \\
$\theta_{i, k}$ & User $i$ 's Shapley value in slot $k$ \\
$\hat{\theta}_{i, k}$ & User $i$ 's approximate Shapley value in slot $k$ \\
$o, \phi(\mathbb{S})$ & One permutation and set of permutations of $\mathbb{S}$ \\
pre $e_{i}(o)$ & User $i$ 's predecessor in the permutation $o$ \\
\hline \hline
\end{tabular}

\section{QuAlity And Reputation Estimation}

In this section, we present detailed designs of the quality estimation module. This module takes raw sensing data from the users as input, quantifies the users' data qualities and reputations, and then filters out anomalous data items. Finally, the platform produces the campaign result, which is the estimation of real physical environment.

\section{A. Quality Estimation}

In a time slot $k$, given the set of the users' sensing data $X_{k}=\left\{x_{1, k}, \ldots, x_{n, k}\right\}$, the quality estimation component calculates the users' data qualities $Q_{k}=\left\{q_{1, k}, \ldots, q_{n, k}\right\}$. Since the ground truth is not known, we rely on the observation that the majority of users contribute reliable data, and utilize the data aggregation with highest density as the criterion to measure the users' data qualities.

We treat the set of sensing data $X_{k}$ as a cluster and denote the distance between any two data items $x_{i, k}$ and $x_{j, k}$ by $\operatorname{dist}\left(x_{i, k}, x_{j, k}\right)$. The distance measurement function $\operatorname{dist}()$, specified by the sensing platform, measures the similarity between different data items. It could be their Euclidean distance, cosine distance, or any other specified similarity distance. A smaller distance usually indicates higher similarity, and vice versa. We also define the centroid of the cluster, denoted by $w_{k}$, to be the data point that minimizes the sum of weighted squared distances between $w_{k}$ and each user's data.

$$
w_{k}=\underset{w_{k}}{\operatorname{argmin}} \sum_{i=1}^{n}\left(\operatorname{dist}^{2}\left(w_{k}, x_{i, k}\right) \times q_{i, k}\right) \text {. }
$$

The quality of each user $i$ 's data is measured based on its deviation $d_{i, k}$ from the cluster centroid, shown in Equation (2). Intuitively, data with higher quality is in closer proximity to the cluster centroid than lower quality ones, which results in a smaller $d_{i, k}$.

$$
d_{i, k}=\operatorname{dist}^{2}\left(w_{k}, x_{i, k}\right) .
$$

Let $\lambda$ be the sum of deviations, i.e., $\lambda=\sum_{i=1}^{n} d_{i, k}$. We update $q_{i, k}$ using the fixed point iteration [25], [30] based on the following equation:

$$
q_{i, k}=\frac{\frac{1}{\frac{d_{i, k}}{\lambda}+\epsilon}}{\sum_{j=1}^{n} \frac{1}{\frac{d_{j, k}}{\lambda}+\epsilon}},
$$

where $\epsilon$ is a small constant real number. The quality estimation algorithm is presented in Algorithm 1. We note that $q_{i, k}$ is a real number within $(0,1)$ and $\sum_{i=1}^{n} q_{i, k}=1$. Our algorithm converges when each user's quality variation between two consecutive iterations is lower than a pre-defined threshold.

\section{B. Reputation Estimation}

After determining the users' data qualities, we present here the design of reputation estimation component, which utilizes the users' historical quality records to estimate their credibility in a long-term view.

Our reputation estimation is based on the observation that a person's reputation in social situations tends to be built up 

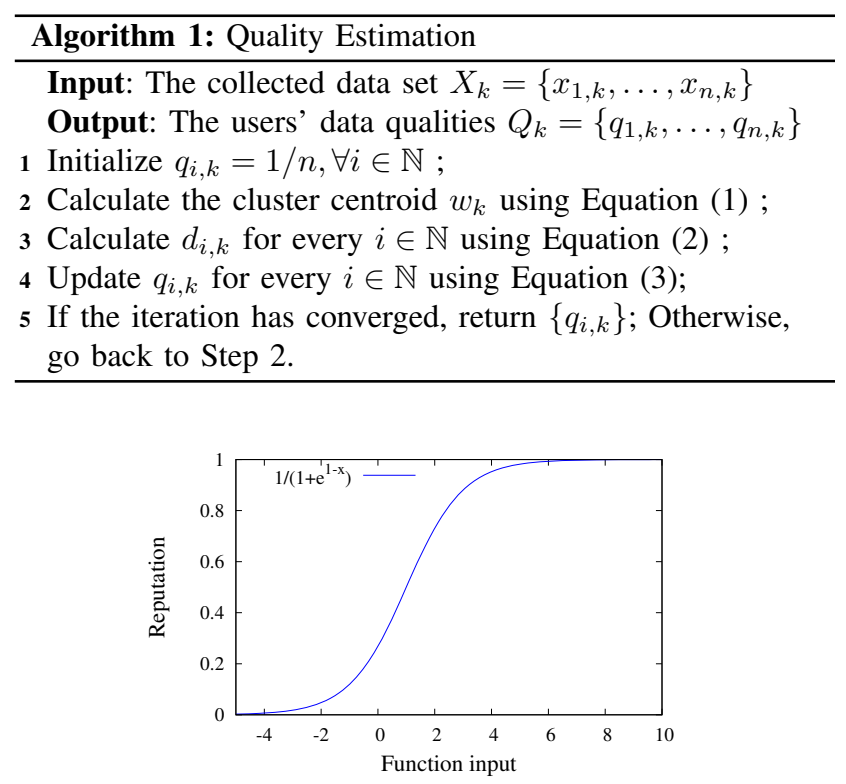

Fig. 3. Logistic Function

gradually after a number of honest behaviors, and can be rapidly torn down after even a few dishonest behaviors [25]. Specifically, we adopt the celebrated logistic function to model this behavior. The generalized logistic function, also known as Richard's curve [38], is formulated below:

$$
R_{i, k}\left(q_{i, k}^{\prime}\right)=A+\frac{B-A}{\left(1+D e^{-F\left(q_{i, k}^{\prime}-M\right)}\right)^{1 / h}},
$$

where $A$ is the lower asymptote, $B$ is the upper asymptote, $D$ depends on the value $R_{i, k}(0), F$ is the growth rate, $M$ determines the maximum growth, and $h$ affects near which asymptote maximum growth occurs. Fig. 3 shows an instance of the logistic function with $A=0, B=1, D=1, F=$ $1, M=1$, and $h=1$.

After each time slot $k$, we update the users' reputations by using the logistic function, whose output $R_{i, k}\left(q_{i, k}^{\prime}\right) \in(0,1)$, is the user $i$ 's updated reputation. The input parameter of the logistic function $q_{i, k}^{\prime}$ is calculated as follows:

$$
q_{i, k}^{\prime}=\sum_{t=1}^{k} \omega^{k-t}\left(q_{i, t}-\frac{1}{n}\right) .
$$

As the formula shows, we aggregate historical information to estimate the users' reputation by summing up all the past quality records, where the exponential term $\omega^{k-t}$, with $0<$ $\omega<1$ being the aging weight, assigns heavier weights to recent records than older ones. The term $q_{i, t}-1 / n$ is used to identify whether user $i$ 's data quality in slot $t$ is above the average, i.e., $q_{i, t}-1 / n>0$ means that the quality of $x_{i, t}$ is above the average and vice versa.

We note that the decrement and increment rates of the users' reputations should be different. One simple approach is to classify the users' behaviors into trustworthy or untrustworthy, and to assign different aging weights to them [25]. We note that this approach assigns users with the same label (i.e., trust- worthy or untrustworthy) the same aging weight. However, in real scenario, the rate of reputation's decrement/increment of a user should be proportional to the degree of the trustworthiness/untrustworthiness of her behavior. For example, a user's reputation should have larger decrement when she contributes "very bad" data than "slightly bad" data. Therefore, we replace $\omega$ with $1-q_{i, t}$, when $q_{i, t} \leq 1 / n$, s.t., the users with lower quality data have higher aging weights and thus results in larger reputation decrements. Similarly, for each user $i$, whose quality is above the average (i.e., $q_{i, t}>1 / n$ ), her aging weight is her quality $q_{i, t}$. We learn from our experiment that the data quality of each user never exceed 0.5 , s.t., the rate of reputation decrement is always larger than the rate of reputation increment.

$$
q_{i, k}^{\prime}= \begin{cases}\sum_{t=1}^{k}\left(1-q_{i, t}\right)^{k-t}\left(q_{i, t}-\frac{1}{n}\right) & \text { if } q_{i, t} \leq \frac{1}{n} \\ \sum_{t=1}^{k} q_{i, t}^{k-t}\left(q_{i, t}-\frac{1}{n}\right) & \text { if } q_{i, t}>\frac{1}{n}\end{cases}
$$

Thus, to determine the user $i$ 's reputation after $k$ slots, we first calculate $q_{i, k}^{\prime}$ using Equation (6) and then apply our reputation function Equation (4). The function output is $i$ 's reputation $R_{i, k}$.

\section{Outlier Detection}

In this subsection, we present an outlier detection technique to find data items that are far away from expectations. For example, the noise readings recorded by a mobile phone that is put in the pocket should be counted as outliers. Specifically, we adopt the concept of distance-based outlier [31], which is a representative method of proximity-based outlier detection.

For the data set $X_{k}$, we define a distance threshold $r$ to be the reasonable neighborhood of a data item. For each data item $x_{i, k} \in X_{k}$, we calculate the number of other data items within the $r$-neighborhood of $x_{i, k}$. If most of the data items are far away from $x_{i, k}$, i.e., not in the $r$-neighborhood of $x_{i, k}$, then $x_{i, k}$ is regarded as an outlier. We present the formal definition below.

Definition 1 (Distance-Based Outlier [31]). Let $r(r \geq 0)$ be the distance threshold and $\mu(0<\mu \leq 1)$ be the fraction threshold. A data object $x_{i, k}$ is $D B(r, \mu)$-outlier if

$$
\frac{\left|\left\{x_{j, k} \mid \operatorname{dist}\left(x_{i, k}, x_{j, k}\right) \leq r\right\}\right|}{\left|X_{k}\right|} \leq \mu .
$$

A simple and efficient algorithm, as shown in Algorithm 2 , is used to classify the users into normal users $\mathbb{N}_{k}^{N}$ and anomalous users $\mathbb{N}_{k}^{A}$. The data items of these anomalous users will be filtered out in the process of generating the estimation of environmental condition.

\section{Campaign Result Generation}

To calculate the environmental estimation in time slot $k$, we first eliminate anomalous data items from collected data set to improve estimation accuracy. Then, we assign each normal data item $x_{i, k}$ a credibility weight $R_{i, k}$, which is user $i$ 's reputation. The reputation-based cluster centroid, calculated 


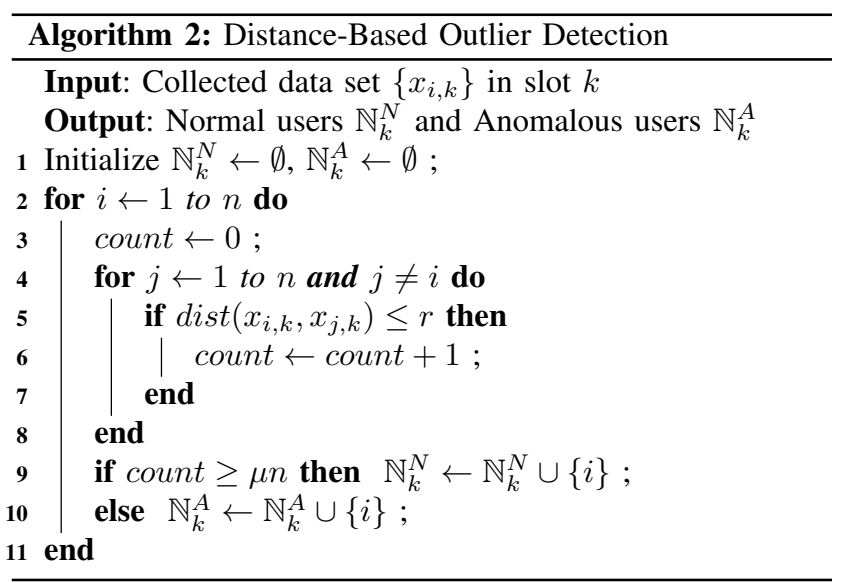

using the equation below, is the campaign estimation for real physical environment in current slot $k$.

$$
\bar{x}_{k}=\underset{\bar{x}_{k}}{\operatorname{argmin}} \sum_{i \in \mathbb{N}_{k}^{N}}\left(\operatorname{dist}^{2}\left(\bar{x}_{k}, x_{i, k}\right) \times R_{i, k}\right) .
$$

We will show in Section VI that the reputation-based cluster centroid achieves superior performance than pure qualitybased one.

\section{SURPLUS SHARING}

To motivate the users' continuous participation, the platform needs to reward each user with a proper amount of payment. However, the platform only has a limited budget. In some existing works, the platform is assumed to be given a fixed budget to run the sensing campaign. While in most of the practical scenarios, especially when the campaign lasts a long period of time (e.g., up to months or years), the platform usually has a dynamic cash flow, which means that the campaign needs to continuously benefit from its real-time estimation. Naturally, the real-time capital inflows, called surplus, is based on the credibility of the generated campaign result.

In this section, we mainly consider the problem of nonfixed surplus sharing, where the total surplus is dynamic and is earned from the real-time campaign result. We first present three desirable properties in designing a good surplus sharing scheme, and discuss several heuristic sharing methods, as well as their limitations. Then, we introduce the concept of Shapley value, and propose a Shapley value-based surplus sharing method. We note that the Shapley value of each user can be considered as the user's contribution to the participatory sensing. Our proposed approach for non-fixed surplus sharing could also be applied in fixed surplus sharing scenarios (by adopting a weighted proportional sharing scheme with each user's Shapley value being her weight).

Formally, the surplus generated by the platform in each slot $k$, called grand surplus, is denoted by $v(\mathbb{N})$, where $\mathbb{N}$ is the set of users and $v: 2^{n} \rightarrow \mathcal{R}$ is the surplus characteristic function. For any subset of users $\mathbb{S} \subseteq \mathbb{N}, v(\mathbb{S})$ represents the surplus earned by the campaign when the set $\mathbb{S}$ of users participate.
We also define user $i$ 's surplus share in slot $k$ as $p_{i, k}$, which is also called $i$ 's payment. The objective of the surplus sharing module is to divide the grand surplus $v(\mathbb{N})$ among the users.

\section{A. Desirable Properties in Surplus Sharing}

In determining each user's surplus share, there are several desirable properties.

Property I: Surplus efficiency. This property indicates that in each time slot, the sum of the users' surplus share should be equal to the grand surplus, i.e., $\sum_{i \in \mathbb{N}} p_{i, k}=v(\mathbb{N})$. In other words, the platform never reserves or overdraws its surplus budget in any time slot.

Property II: Outliers get nothing and normal users all get paid. This property is derived from the two-fold goal of the participatory sensing campaign. On one hand, the platform wishes to penalize untrustworthy behaviors, s.t., users who are classified as outliers in some time slot shall get zero surplus share, since their data is far away from the group consensus and thus makes no meaningful contributions to the campaign in that slot. On the other hand, to motivate the users' continuous participation, every user receives a positive surplus share as long as she is not counted as an outlier. Formally, if $i \in \mathbb{N}_{k}^{A}$, then $p_{i, k}=0$; otherwise $p_{i, k}>0$.

Property III: Monotonic rule. It means that for any two normal users, the one with higher reputation should gain more surplus than the other one. This rule indicates the fairness of surplus sharing, i.e., users' rewards are proportional to the qualities of their contributions. Formally, in any slot $k$, for any two users $i, j \in \mathbb{N}_{k}^{N}$, if $R_{i, k}>R_{j, k}$, then $p_{i, k}>p_{j, k}$, and if $R_{i, k}=R_{j, k}$, then $p_{i, k}=p_{j, k}$.

\section{B. Several Heuristic Sharing Methods}

One simple surplus sharing approach is equal share, i.e., to assign each user an equal share of the total surplus $p_{i, k}=v(\mathbb{N}) / n$. However, this allocation rule suffers from a serious fairness issue, i.e., users with low quality data earn the same rewards as those who made high quality contributions, which may drive the latter group to leave the campaign or to contribute low quality data.

Another approach is individual surplus contribution, which assigns each user $i$ with the amount of surplus that the campaign generates when only $i$ participates, i.e., $p_{i, k}=v(\{i\})$. This approach takes the users' data qualities and reputations into surplus calculation, and thus satisfies monotone rule. However, it cannot guarantee the surplus efficiency, since the sum of allocated surplus may not be equal to the surplus budget, i.e., $\sum_{i \in \mathbb{N}} p_{i, k} \neq v(\mathbb{N})$.

The third heuristic sharing method is called marginal surplus contribution. It states that the surplus share of each user $i$ is the difference between total surplus when $i$ participates and when $i$ does not participate, given all other conditions remain the same. Formally, $p_{i, k}=v(\mathbb{N})-v(\mathbb{N} \backslash\{i\})$. This approach also violates the surplus efficiency, i.e., $\sum_{i \in \mathbb{N}} p_{i, k} \neq v(\mathbb{N})$. 


\section{Shapley Value}

Considering the limitations of the previously mentioned heuristic methods, we present an alternative Shapley valuebased approach, which can achieve all the three desirable properties.

Definition 2 (Shapley Value [26], [37]). In surplus sharing, the Shapley value of $i$ is defined by

$$
\theta_{i, k}(\mathbb{N}, v)=\sum_{\mathbb{S} \subseteq \mathbb{N} \backslash\{i\}} \frac{[v(\mathbb{S} \cup\{i\})-v(\mathbb{S})]|\mathbb{N}| !}{|\mathbb{S}| !(|\mathbb{N}|-|\mathbb{S}|-1) !},
$$

where $|\mathbb{S}|$ and $|\mathbb{N}|$ are the cardinality of $\mathbb{S}$ and $\mathbb{N}$ respectively.

The Shapley value is calculated by taking a random permutation of users from the set of all $n$ ! possible permutations, and allocating each user her expected marginal surplus contribution in this permutation. It has been proved that the Shapley value is the unique value satisfying the following four axioms [26].

Axiom 1 (Efficiency). $\sum_{i \in \mathbb{N}} \theta_{i, k}=v(\mathbb{N})$.

Axiom 2 (Symmetry). If $\forall \mathbb{S} \subseteq \mathbb{N} \backslash\{i, j\}, v(\mathbb{S} \cup\{i\})=v(\mathbb{S} \cup$ $\{j\})$, then $\theta_{i, k}=\theta_{j, k}$.

Axiom 3 (Dummy). If $\forall \mathbb{S} \subseteq \mathbb{N} \backslash\{i\}, v(\mathbb{S} \cup\{i\})=v(\mathbb{S})$, then $\theta_{i, k}=0$.

Axiom 4 (Additivity). For any two surplus function $v_{1}$ and $v_{2}, \theta_{i, k}\left(v_{1}\right)+\theta_{i, k}\left(v_{2}\right)=\theta_{i, k}\left(v_{1}+v_{2}\right), \forall i \in \mathbb{N}$.

The efficiency axiom states that the sum of the users' surplus share should be equal to the grand surplus, which matches the property of the surplus efficiency in Section V-A. The symmetry axiom indicates that two users having equal marginal surplus contributions should receive the same amount of surplus share. The dummy axiom says that a user who does not contribute to surplus generation should receive nothing, i.e., outliers receive zero surplus share. These two axioms satisfy the requirements of our second and third desirable property respectively. The additivity axiom means that combining two games into one, each user's received surplus share remains the same. In our setting, the additivity says that the total revenue received by any user in the long period campaign should be equal to the sum of her surplus share gained in every single slot.

We note that the four axioms are inherent properties of our surplus sharing. With a well-defined surplus function, e.g., $v(\mathbb{S})$ is monotone increasing subject to $\sum_{i \in \mathbb{S}} R_{i, k}$, we can guarantee that a user with a higher reputation has a larger Shapley value than lower reputation one, since she contributes more to the marginal surplus $v(\mathbb{S} \cup\{i\})-v(\mathbb{S})$. Besides, if a user's marginal contribution is positive, then her Shapley value is positive. We present an instance of surplus function in Section VI-A.

Our Shapley value-based surplus sharing rule is presented below. In each slot, the payments of anomalous users are zero, while the payment of each normal user is her Shapley value with $\mathbb{N}_{k}^{N}$ being the grand coalition.

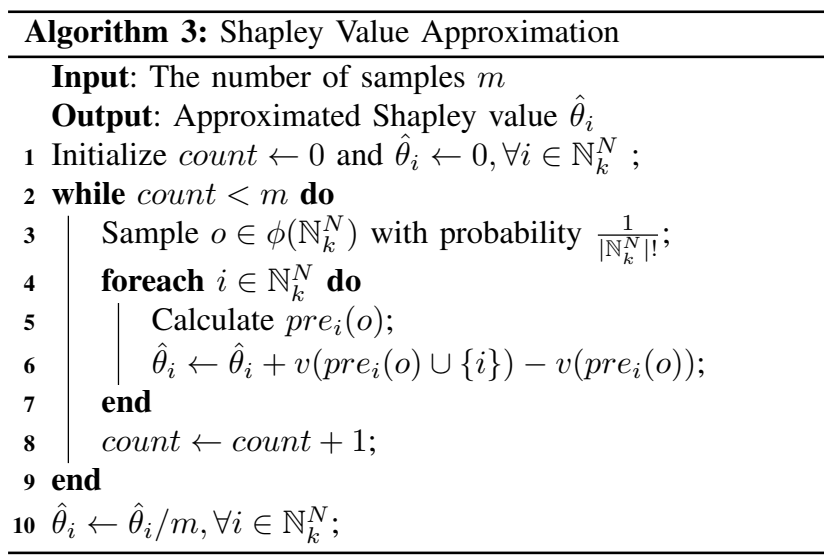

$$
p_{i, k}= \begin{cases}0 & \text { if } i \in \mathbb{N}_{k}^{A} \\ \theta_{i, k}\left(\mathbb{N}_{k}^{N}, v\right) & \text { if } i \in \mathbb{N}_{k}^{N}\end{cases}
$$

\section{Approximate Shapley Value}

Due to the appealing properties of Shapley value and its excellent match for our model, we reward each normal user with the surplus share of her Shapley value. However, we observe that the number of subset of $\mathbb{N}_{k}^{N}$ is exponential to its cardinality, therefore the calculation of Shapley value involves an exponential time complexity. When the number of normal users is large, this approach would be impractical. To settle this computational infeasibility, we propose an efficient approximation of the Shapley value based on random samping.

Let $\phi\left(\mathbb{N}_{k}^{N}\right)$ denote the set of all $\left|\mathbb{N}_{k}^{N}\right|$ ! permutations of $\mathbb{N}_{k}^{N}$, and $\hat{\theta}_{i}$ represent the approximated Shapley value of user $i$. For any sampled permutation $o \in \phi\left(\mathbb{N}_{k}^{N}\right)$, the set of users appeared before $i$ is defined as the predecessors of $i$, denoted by $\operatorname{pre}_{i}(o)$. For example, a sampled permutation is shown below, as well as $i$ 's and $j$ 's predecessors.

$$
\underbrace{\overbrace{1,2, \cdots, i-1}^{\text {i's predecessor }}, i, i+1, \cdots, j-1}_{\text {j's predecessor }}, j, j+1, \cdots,\left|\mathbb{N}_{k}^{N}\right|
$$

Our proposed algorithm, as shown in Algorithm 3, randomly selects $m$ samples from $\phi\left(\mathbb{N}_{k}^{N}\right)$ with equal probability. For each sampled permutation $o \in \phi\left(\mathbb{N}_{k}^{N}\right)$, we calculate the predecessor of the each user $i$. Then, the algorithm iteratively sums up each user's marginal contributions over the predecessors of each sample. The estimated Shapley value will be the average of the marginal contributions over the samples. The payment of each user $i$ in time slot $k$ is shown below:

$$
p_{i, k}= \begin{cases}0 & \text { if } i \in \mathbb{N}_{k}^{A}, \\ \hat{\theta}_{i, k}\left(\mathbb{N}_{k}^{N}, v\right) & \text { if } i \in \mathbb{N}_{k}^{N} .\end{cases}
$$

We note that the complexity of our proposed algorithm is $O\left(m\left|\mathbb{N}_{k}^{N}\right|\right)$, which is in polynomial time. In addition, it can be proved that the approximate Shapley value also satisfies all the four axioms of the original Shapley value. Due to limitations of space, we omit the proof in this paper. 


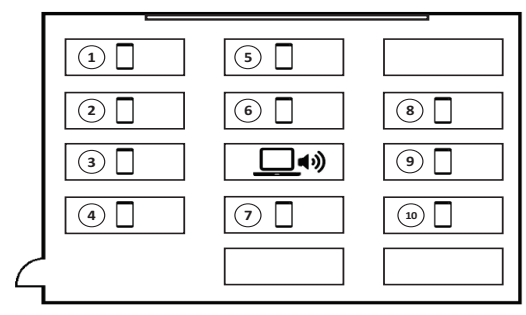

Fig. 4. Device Deployment

\section{EVALUATIONS}

In this section, we conduct a participatory sensing experiment to evaluate our proposed methods. We first describe our experiment setup in Section VI-A, and then present experiment results in Section VI-B and Section VI-C.

\section{A. Experiment Setup}

We consider a noise monitoring participatory sensing application, where mobile devices are required to measure their ambient noise level and then the platform generates the noise estimation based on the collected sensing readings.

In our experiments, 10 mobile devices are deployed to act as participatory sensing users, including 5 first-generation Google Nexus 7 tablets (D1 to D5) and 5 second-generation Google Nexus 7 tablets (D6 to D10). All of them are carried with Android 4.4.3 operating system. The ambient noise is measured and recorded by an off-the-shelf application, called NoiseTube [39], which samples the acoustic signal and calculates the sound level every second in decibel (dB). Our experiment is conducted in a $10 \mathrm{~m} \times 8 \mathrm{~m}$ room to ensure that the sound attenuation in distance is trivial. A computer, which continuously plays movies, serves as the noise source and is placed in the center of the room. Mobile devices are deployed around the computer as shown in Fig 4. We also deploy a WENSN WS1361 decibel meter to measure the ground truth.

Recall that our objective is to estimate the users' data qualities and characterize their long-term behaviors. According to the real life experiences, we artificially create situations where users may adopt incorrect sensing approaches and have various behaviors. In noise monitoring application, the correct measurement approach is to expose the mobile device directly to air. However, in real scenarios, users may intentionally or unintentionally take the wrong measurement approaches, e.g., placing the phone in a pocket or bag, which may blemish their data qualities. To simulate these differences, in our experiment, most devices take the proper sensing method, while some devices are covered by clothes or put into a bag to simulate incorrect approaches. For simplicity, we refer " 1 " to the correct measurement approach and " 0 " to incorrect ones. Besides, we divide users into several categories and assign each category a specific sensing behavior, shown in Table II. In our setting, device D3 and D9 are strictly obedient to the platform's instructions and always expose their mobile devices to air. Device D1 and D7 are rigorous in performing the sensing task correctly, but there are 10\% 20\% unavoidable time when they have to put their devices into pockets or bags.
TABLE II

USER BEHAVIOR CLASSIFICATION

\begin{tabular}{lll}
\hline Category & Device & Description \\
\hline Obedient user & 3,9 & always being "1" \\
Rigorous user & 1,7 & with $80 \% \sim 90 \%$ possibility being "1" \\
Careless user & $5,8,10$ & with $60 \% \sim 70 \%$ possibility being "1" \\
Indifferent user & 4 & with $50 \%$ possibility being "1" \\
Malicious user & 2,6 & with $<10 \%$ possibility being "1" \\
\hline
\end{tabular}

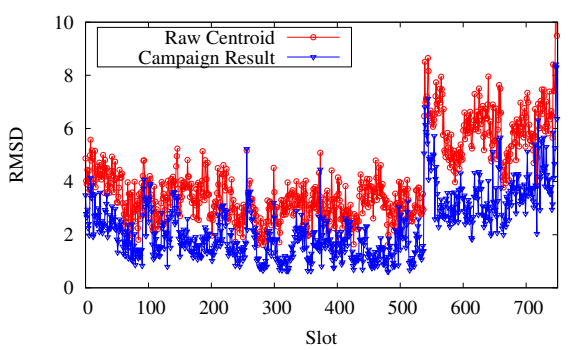

(a) Raw Centroid vs Campaign Result

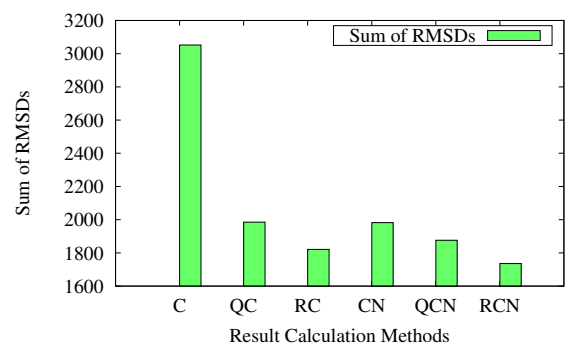

(b) Different Sensing Results

Fig. 5. Result Comparison

The category with largest number of users is careless and we assume careless users have $60 \% \sim 70 \%$ being " 1 ". Device 4 is indifferent of the sensing task and he places his device into or out of his pocket any time he wants, thus with half percentage being "1". Malicious users, such as D2 and D6, deliberately contribute erroneous data in most of the time.

Our experiment lasts 750 minutes with the slot duration being 1 minute, and collects over 450,000 data items in total. Based on user behavior classification, we manually change the measuring approaches of the devices (either exposed to air or covered by clothes) with their predefined possibilities. For instance, for device D8, we reset its sensing approach every 15 minutes with $60 \% \sim 70 \%$ possibility exposed to air and $30 \% \sim 40 \%$ covered by clothes. Detailed deployment settings and graphical presentations of sensing data are provided in our technical report [40]. It can be clearly observed that the detected noise level with the device exposed is about $5 \mathrm{~dB}$ higher than covered.

In the quality estimation module, we adopt the Euclidean distance to measure the similarity between any two data items. We note that in the noise monitoring scenario, each user's sensing reading in any slot is a vector consisting 60 elements (since the slot duration is 60 seconds) and thus the arithmetic operations used in quality estimation are correspondingly vector operations. The $\epsilon$ used in Equation (3) is 0.01 and we 

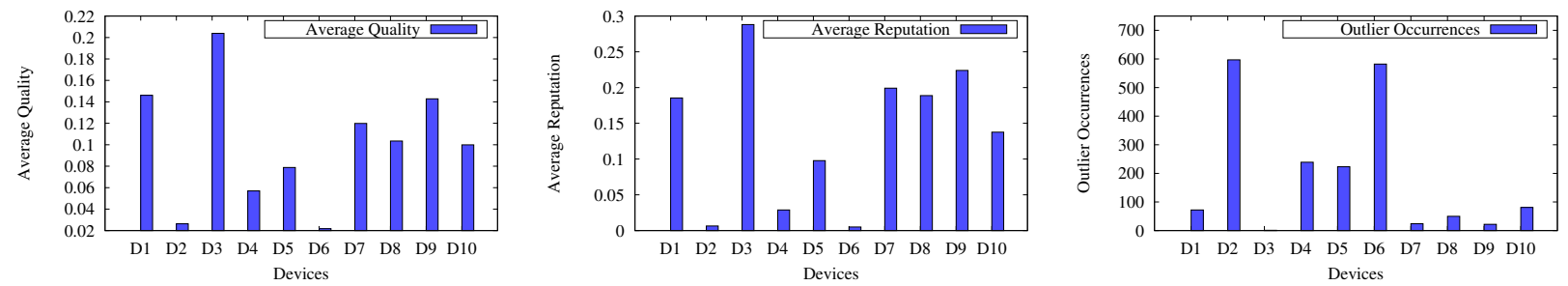

Fig. 6. Qualities, reputations, and outlier occurrences of devices

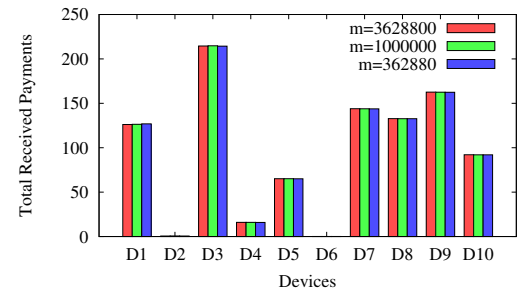

(a) Total Payment of Each Device

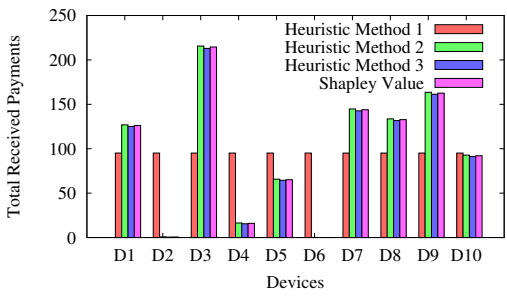

(b) Total Payment of Different Methods

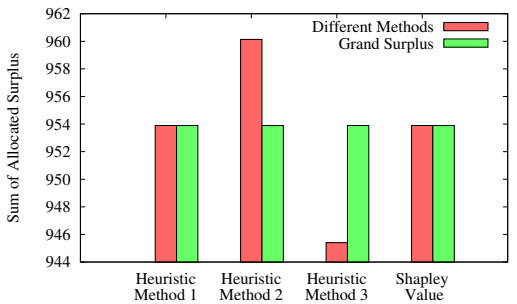

(c) Surplus Efficiency

Fig. 7. Comparisons of different surplus sharing methods

iterate our quality estimation algorithm for 10,000 times each slot. The parameters for the generalized logistic function are: $A=0, B=1, D=1, F=1, M=1$, and $h=1$. In outlier detection, the default distance threshold $r$ and fraction threshold $\mu$ are 4 and 0.31 respectively.

In surplus sharing module, we apply the same surplus function below to every sensing slot:

$$
v(\mathbb{S})=V \times \frac{g(|\mathbb{S}|) \sum_{i \in \mathbb{S}} R_{i, k}}{|\mathbb{S}|},
$$

where $V=1000$ is the scaling factor and $g(n)=n /(n+1000)$ is the Bühlmann credibility function [41], which is widely used in credibility theory to model the relationship between the number of users and the credibility of the user-generated results.

\section{B. Experiment Results of Quality Estimation}

Recall that our sensing result is generated by finding the Reputation-weighted Centroid of the Normal user cluster, denoted by RCN, i.e., the distance is weighted with reputation and the cluster is formed by normal users, as Equation (7). Similarly, we define QCN (quality-weighted centroid of normal users), CN (raw centroid of normal users), RC (reputationweighted centroid of users), QC (quality-weighted centroid of users) and $\mathrm{C}$ (raw centroid of users). Mathematical definitions are provided in our technical report [40].

We compare our sensing results with the ground truth by adopting the Root Mean Square Deviation (RMSD). For any given data vector $x_{i, k}$, the RMSD is defined as $\sqrt{\text { dist }^{2}\left(x_{i, k}, \hat{x}_{k}\right) / T}$, where $\hat{x}_{k}$ is the ground truth in slot $k$. Fig. 5(a) shows the RMSDs of raw centroid (C) and our campaign result $(\mathrm{RCN})$ in every slot. We can see that the RMSD of RCN is about $50 \%$ lower than the raw centroid. To get a complete comparisons of different campaign result calculation methods, for each method, we sum up its RMSDs of the total 750 slots, and provide the results in Fig. 5(b).
We observe that under the same cluster (either $\mathbb{N}$ or $\mathbb{N}_{k}^{N}$ ), the reputation-weighted centroid always results in lowest sum of RMSDs, while the raw centroid the highest. That is because that the reputation-based methods completely characterize the users' credibility, while raw centroid methods do not consider the quality differences of collected data and treat all the users equally. The quality-weighted centroid methods involve data qualities in their result calculation, but they never take the users' long-term reputations into consideration. That is why QC's and QCN's sum of RMSDs are lower than raw centroid methods $(\mathrm{C}$ and $\mathrm{CN})$ but higher than reputationweighted ones ( $\mathrm{RC}$ and $\mathrm{RCN}$ ). It can also be observed that sensing results calculated using normal-user cluster $\mathbb{N}_{k}^{N}$ results in lower sum of RMSDs than user cluster $\mathbb{N}$, which indicates that eliminating anomalous data items improves the accuracy of the campaign result.

Fig. 6 presents the comparisons of qualities, reputation$\mathrm{s}$, and outlier occurrences of all the users, where quality and reputation are measured using their average value, i.e., $\sum_{k=1}^{750} q_{i, k} / 750$ and $\sum_{k=1}^{750} R_{i, k} / 750$ respectively. The outlier occurrence of a user is the number of times when she is counted as an outlier. We observe that the users' qualities and reputations are proportional to the level of their obedience, while the outlier occurrences are inversely proportional to that, which aligns to our user behavior classification. For example, the obedient users (D3 and D9) have the highest qualities, highest reputations, and fewest outlier occurrences. Rigorous users (D1 and D7) have the second highest qualities/reputations and second lowest outlier occurrences. Malicious users (D2 and D6) receive approximately zero qualities and reputation scores, with outlier occurrences over $80 \%$ of the total slots.

\section{Experiment Results of Surplus Sharing}

Fig. 7(a) shows the total payment received by each devices, i.e., $\sum_{k=1}^{750} p_{i, k}$, where $m$ is the number of permutations sampled. We note that $m=10 !=3,628,800$ is the original 
Shapley value calculation method, while $m=1,000,000$ and 362,880 are both approximate ones. We observe that the users' total payments are nearly the same under different values of $m$, which indicates that the approximate Shapley value also satisfies all the four properties of the original Shapley value. Besides, each user's total payment is proportional to the quality of her contribution. For example, obedient users (D3 and D9) receive the most payments, while malicious users (D2 and D6) receive nearly zero payments.

We also compare the performance of Shapley value with three heuristic methods mentioned in Section V-B, which are equal share, individual contribution, and marginal contribution respectively. Fig. 7(b) compares the total received payments by each user under different surplus sharing methods, and Fig. 7 (c) compares the sum of allocated surplus $\sum_{i=1}^{n} \sum_{k=1}^{750} p_{i, k}$ with the grand surplus. We can see that the equal share method satisfies surplus efficiency, but violates the second and third desirable properties in Section V-A, since it never considers the users' data qualities. The other two heuristic methods have the similar surplus distribution patterns as Shapley value, but they violate the surplus efficiency.

\section{CONCLUSION}

In this paper, we have considered the quality-base surplus sharing problem for participatory sensing. We have proposed a method to quantify users' data qualities and reputations, and have applied an outlier detection technique to improve estimation accuracy. Based on the cooperative game model, we have proposed an approximate Shapley value-based method to determine users' surplus shares. Our experiment results have shown that our approach achieves good performance in terms of both quality estimation and surplus sharing.

\section{REFERENCES}

[1] J. A. Burke, D. Estrin, M. Hansen, A. Parker, N. Ramanathan, S. Reddy, and M. B. Srivastava, "Participatory sensing," Center for Embedded Network Sensing, 2006.

[2] N. D. Lane, E. Miluzzo, H. Lu, D. Peebles, T. Choudhury, and A. T. Campbell, "A survey of mobile phone sensing," Communications Magazine, IEEE, vol. 48, no. 9, pp. 140-150, 2010.

[3] R. K. Ganti, F. Ye, and H. Lei, "Mobile crowdsensing: current state and future challenges," Communications Magazine, IEEE, vol. 49, no. 11, pp. 32-39, 2011.

[4] M. Azizyan, I. Constandache, and R. Roy Choudhury, "Surroundsense: mobile phone localization via ambience fingerprinting," in MobiCom, 2009.

[5] Z. Yang, C. Wu, and Y. Liu, "Locating in fingerprint space: wireless indoor localization with little human intervention," in MobiCom, 2012.

[6] A. Rai, K. K. Chintalapudi, V. N. Padmanabhan, and R. Sen, "Zee: zero-effort crowdsourcing for indoor localization," in MobiCom, 2012.

[7] S. Yang, P. Dessai, M. Verma, and M. Gerla, "Freeloc: Calibration-free crowdsourced indoor localization," in INFOCOM, 2013.

[8] M. Alzantot and M. Youssef, "Crowdinside: automatic construction of indoor floorplans," in SIGSPATIAL, 2012.

[9] R. Gao, M. Zhao, T. Ye, F. Ye, Y. Wang, K. Bian, T. Wang, and X. Li, "Jigsaw: Indoor floor plan reconstruction via mobile crowdsensing," in MobiCom, 2014.

[10] M. Mun, S. Reddy, K. Shilton, N. Yau, J. Burke, D. Estrin, M. Hansen, E. Howard, R. West, and P. Boda, "PEIR, the personal environmental impact report, as a platform for participatory sensing systems research," in MobiSys, 2009.

[11] A. Thiagarajan, L. Ravindranath, K. LaCurts, S. Madden, H. Balakrishnan, S. Toledo, and J. Eriksson, "Vtrack: accurate, energy-aware road traffic delay estimation using mobile phones," in SenSys, 2009.
[12] R. K. Ganti, N. Pham, H. Ahmadi, S. Nangia, and T. F. Abdelzaher, "Greengps: a participatory sensing fuel-efficient maps application," in MobiSys, 2010.

[13] P. Zhou, Y. Zheng, and M. Li, "How long to wait?: predicting bus arrival time with mobile phone based participatory sensing," in MobiSys, 2012.

[14] S. Nawaz, C. Efstratiou, and C. Mascolo, "Parksense: a smartphone based sensing system for on-street parking," in MobiCom, 2013.

[15] Y. Wang, X. Liu, H. Wei, G. Forman, C. Chen, and Y. Zhu, "Crowdatlas: Self-updating maps for cloud and personal use," in MobiSys, 2013.

[16] Y. Wang, W. Hu, Y. Wu, and G. Cao, "Smartphoto: a resourceaware crowdsourcing approach for image sensing with smartphones," in MobiHoc, 2014.

[17] J.-S. Lee and B. Hoh, "Sell your experiences: a market mechanism based incentive for participatory sensing," in PerCom, 2010.

[18] D. Yang, G. Xue, X. Fang, and J. Tang, "Crowdsourcing to smartphones: incentive mechanism design for mobile phone sensing," in MobiCom, 2012.

[19] I. Koutsopoulos, "Optimal incentive-driven design of participatory sensing systems," in INFOCOM, 2013.

[20] D. Zhao, X.-Y. Li, and H. Ma, "How to crowdsource tasks truthfully without sacrificing utility: Online incentive mechanisms with budget constraint," in INFOCOM, 2014

[21] M. Dong, T. Lan, and L. Zhong, "Rethink energy accounting with cooperative game theory," in MobiCom, 2014.

[22] Y. Zhang and M. van der Schaar, "Reputation-based incentive protocols in crowdsourcing applications," in INFOCOM, 2012.

[23] L. G. Jaimes, I. Vergara-Laurens, and M. A. Labrador, "A location-based incentive mechanism for participatory sensing systems with budget constraints," in PerCom, 2012.

[24] T. Luo, H.-P. Tan, and L. Xia, "Profit-maximizing incentive for participatory sensing," in INFOCOM, 2014.

[25] K. L. Huang, S. S. Kanhere, and W. Hu, "Are you contributing trustworthy data?: the case for a reputation system in participatory sensing," in MSWiM, 2010.

[26] L. S. Shapley, "A value for n-person games," DTIC Document, Tech. Rep., 1952.

[27] A. E. Roth, The Shapley value: essays in honor of Lloyd S. Shapley. Cambridge University Press, 1988.

[28] J. Han and M. Kamber, "Data mining: concepts and techniques," Morgan Kaufmann, vol. 5, 2001.

[29] S. Ganeriwal, L. K. Balzano, and M. B. Srivastava, "Reputation-based framework for high integrity sensor networks," ACM Transactions on Sensor Networks (TOSN), vol. 4, no. 3, p. 15, 2008.

[30] C. T. Chou, A. Ignjatovic, and W. Hu, "Efficient computation of robust average of compressive sensing data in wireless sensor networks in the presence of sensor faults," IEEE Transactions on Parallel and Distributed Systems, vol. 24, no. 8, pp. 1525-1534, 2013.

[31] E. M. Knox and R. T. Ng, "Algorithms for mining distance-based outliers in large datasets," in $V L D B, 1998$.

[32] M. M. Breunig, H.-P. Kriegel, R. T. Ng, and J. Sander, "Lof: identifying density-based local outliers," in ACM Sigmod Record, vol. 29, no. 2. ACM, 2000, pp. 93-104.

[33] V. Misra, S. Ioannidis, A. Chaintreau, and L. Massoulié, "Incentivizing peer-assisted services: a fluid shapley value approach," in SIGMETRICS, 2010 .

[34] R. Narayanam and Y. Narahari, "A shapley value-based approach to discover influential nodes in social networks," IEEE Transactions on Automation Science and Engineering, no. 99, pp. 1-18, 2010.

[35] R. T. Ma, D. M. Chiu, J. Lui, V. Misra, and D. Rubenstein, "Internet economics: The use of shapley value for isp settlement," IEEE/ACM Transactions on Networking (TON), vol. 18, no. 3, pp. 775-787, 2010.

[36] S. He, D.-H. Shin, J. Zhang, and J. Chen, "Toward optimal allocation of location dependent tasks in crowdsensing," in INFOCOM, 2014.

[37] N. Nisan, T. Roughgarden, E. Tardos, and V. V. Vazirani, Algorithmic game theory. Cambridge University Press, 2007.

[38] F. Richards, "A flexible growth function for empirical use," Journal of experimental Botany, vol. 10, no. 2, pp. 290-301, 1959.

[39] (2008) Noisetube. [Online]. Available: http://www.noisetube.net

[40] S. Yang, F. Wu, S. Tang, X. Gao, B. Yang, and G. Chen. (2015, Mar.) A quality-based surplus sharing method for participatory sensing. Technical Report. [Online]. Available: https://www.dropbox.com/s/h4tf42vv999jn7d/report.pdf?dl=0

[41] H. Bühlmann, "Experience rating and credibility," Astin Bulletin, vol. 4, no. 03, pp. 199-207, 1967. 